\title{
Video Article \\ Monitoring the Reductive and Oxidative Half-Reactions of a Flavin-Dependent Monooxygenase using Stopped-Flow Spectrophotometry
}

\author{
Elvira Romero ${ }^{1}$, Reeder Robinson ${ }^{1}$, Pablo Sobrado ${ }^{1}$ \\ ${ }^{1}$ Department of Biochemistry, Virginia Polytechnic Institute and State University \\ Correspondence to: Pablo Sobrado at psobrado@vt.edu \\ URL: https://www.jove.com/video/3803 \\ DOI: doi:10.3791/3803
}

Keywords: Bioengineering, Issue 61, Stopped-flow, kinetic mechanism, SidA, C4a-hydroperoxyflavin, monooxygenase, Aspergillus fumigatus Date Published: 3/18/2012

Citation: Romero, E., Robinson, R., Sobrado, P. Monitoring the Reductive and Oxidative Half-Reactions of a Flavin-Dependent Monooxygenase using Stopped-Flow Spectrophotometry. J. Vis. Exp. (61), e3803, doi:10.3791/3803 (2012).

\section{Abstract}

Aspergillus fumigatus siderophore $\mathrm{A}(\mathrm{Sid} \mathrm{A})$ is an $\mathrm{FAD}$-containing monooxygenase that catalyzes the hydroxylation of ornithine in the biosynthesis of hydroxamate siderophores that are essential for virulence (e.g. ferricrocin or $N^{\prime}, N^{\prime \prime}, N^{\prime \prime}$--triacetylfusarinine $\left.\mathrm{C}\right)^{1}$. The reaction catalyzed by SidA can be divided into reductive and oxidative half-reactions (Scheme 1). In the reductive half-reaction, the oxidized FAD bound to $A f \mathrm{SidA}$, is reduced by NADPH ${ }^{2,3}$. In the oxidative half-reaction, the reduced cofactor reacts with molecular oxygen to form a C4ahydroperoxyflavin intermediate, which transfers an oxygen atom to ornithine. Here, we describe a procedure to measure the rates and detect the different spectral forms of SidA using a stopped-flow instrument installed in an anaerobic glove box. In the stopped-flow instrument, small volumes of reactants are rapidly mixed, and after the flow is stopped by the stop syringe (Figure 1), the spectral changes of the solution placed in the observation cell are recorded over time. In the first part of the experiment, we show how we can use the stopped-flow instrument in single mode, where the anaerobic reduction of the flavin in Af SidA by NADPH is directly measured. We then use double mixing settings where Af SidA is first anaerobically reduced by NADPH for a designated period of time in an aging loop, and then reacted with molecular oxygen in the observation cell (Figure 1). In order to perform this experiment, anaerobic buffers are necessary because when only the reductive half-reaction is monitored, any oxygen in the solutions will react with the reduced flavin cofactor and form a C4a-hydroperoxyflavin intermediate that will ultimately decay back into the oxidized flavin. This would not allow the user to accurately measure rates of reduction since there would be complete turnover of the enzyme. When the oxidative half-reaction is being studied the enzyme must be reduced in the absence of oxygen so that just the steps between reduction and oxidation are observed. One of the buffers used in this experiment is oxygen saturated so that we can study the oxidative half-reaction at higher concentrations of oxygen. These are often the procedures carried out when studying either the reductive or oxidative half-reactions with flavin-containing monooxygenases. The time scale of the pre-steady-state experiments performed with the stopped-flow is milliseconds to seconds, which allow the determination of intrinsic rate constants and the detection and identification of intermediates in the reaction ${ }^{4}$. The procedures described here can be applied to other flavin-dependent monooxygenases. ${ }^{5,6}$

\section{Video Link}

The video component of this article can be found at https://www.jove.com/video/3803/

\section{Preparation of Anaerobic Buffer}

1. Prepare $1 \mathrm{~L}$ of $100 \mathrm{mM}$ potassium phosphate buffer, $\mathrm{pH}$ 7.5. Pour $250 \mathrm{~mL}$ of the buffer into a $500-\mathrm{mL}$ Büchner flask with a stir bar.

2. Seal the flask with a rubber stopper and place it on a stir plate. Connect the short tube of the flask to a Schlenk line.

3. Degas the buffer under vacuum for 5 hours at room temperature with agitation. During this time period, perform 5 consecutive rounds of vacuum degassing and flushing with argon every hour.

4. Flush the flask with argon for 10 seconds and disconnect it from the vacuum manifold. Place the flask inside the glove box

5. Leave the flask open in the glove box overnight with vigorous agitation.

\section{Removing Oxygen from the Stopped-flow System}

1. Prepare $1 \mathrm{~L}$ of $0.1 \mathrm{M}$ sodium acetate, $\mathrm{pH}$ 5.0. Pour $125 \mathrm{~mL}$ of this buffer into a $250-\mathrm{mL}$ Büchner flask and perform steps $1.2-1.4$.

2. Weigh out $5 \mathrm{mg}$ glucose oxidase from Aspergillus niger $(181300 \mathrm{U} / \mathrm{g})$ and $0.9 \mathrm{~g}$ glucose using a $1.5 \mathrm{~mL}$ Eppendorf tube and $50 \mathrm{~mL}$ Falcon conical tube, respectively. Place both containers into the glove box.

3. Dissolve glucose oxidase and glucose in $50 \mathrm{~mL}$ of anaerobic $0.1 \mathrm{M}$ sodium acetate, $\mathrm{pH} 5.0$ (final concentrations of $18.13 \mathrm{U} / \mathrm{mL}$ and $100 \mathrm{mM}$, respectively). Fill the reservoir syringes with this solution (Figure 1).

4. Ensure that the drive valves are in the "load" position and fill the drive syringes (Figure 1). Turn the F valve to the "drive" position. Empty the stop syringe by clicking on Empty in the Pro-Data control software. Push the buffer from drive syringe $\mathrm{F}$ through the flow circuit by manually 
raising the corresponding drive ram. Repeat this step ten times in total. Perform the same procedure with the other three drive syringes. Wait one hour.

5. Replace the solution of the reservoir syringes with the same buffer containing glucose oxidase and glucose. Repeat step 2.4 .

6. Repeat step 2.5 and allow the solution to stand in the flow system overnight.

7. After overnight incubation, repeat step 2.5.

\section{Preparation of Oxygen Saturated Buffer}

1. Prepare $100 \mathrm{mM}$ potassium phosphate buffer $(\mathrm{pH} 7.5)$ and pour $50 \mathrm{~mL}$ into a $50-\mathrm{mL}$ vial with a stir bar. Cap the vial with a Wheaton stopper and a Wheaton aluminum seal.

2. Place the vial on ice. Immerse a long needle connected to a tank containing $100 \%$ oxygen into the solution. Insert another short needle through the stopper of the vial as a vent.

3. Bubble the solution with $100 \%$ oxygen for 1 hour with agitation.

4. First remove the short needle from the vial and wait 10 seconds before removing the long needle. Place the closed vial in the glove box.

\section{Preparation of NADPH Solution}

1. Weigh out $1 \mathrm{mg}$ of NADPH in a $1.5 \mathrm{~mL}$ Eppendorf tube and place it into the glove box

2. Dissolve the NADPH in $300 \mu \mathrm{L}$ of anaerobic $100 \mathrm{mM}$ potassium phosphate buffer, $\mathrm{pH}$ 7.5. Remove $30 \mu \mathrm{L}$ of this solution from the glove box to determine the NADPH concentration with a spectrophotometer $\left(\varepsilon_{340 \mathrm{~nm}}=6,270 \mathrm{M}^{-1} \mathrm{~cm}^{-1}\right)$.

\section{Removal of Oxygen from the Enzyme Solution}

1. Take $400 \mu \mathrm{L}$ of enzyme stock solution from the freezer and thaw in the glove box. The enzyme stock solution ( $360 \mu \mathrm{M})$ was previously prepared in $100 \mathrm{mM}$ potassium phosphate buffer $(\mathrm{pH} 7.5)$ containing $100 \mathrm{mM} \mathrm{NaCl}$, and frozen in liquid nitrogen.

2. Transfer the enzyme solution into a $25-\mathrm{mL}$ vial with a stir bar, cap the vial with a Wheaton stopper and a Wheaton aluminum seal and remove from the glove box.

3. Place the vial on ice and connect it to a Schlenk line by inserting a needle through the stopper of the vial.

4. Degas the enzyme solution by performing 5 consecutive rounds of vacuum degassing and flushing with argon every 20 minutes for 1 hour.

5. Flush the vial with argon for 10 seconds and disconnect it from the vacuum manifold. Place the vial on ice inside the glove box.

\section{Reductive Half-reaction: Monitoring Flavin Reduction}

1. Prepare the stopped-flow instrument and the Pro-Data control software for single mixing mode following the manufacturer's protocol.

2. Turn on a circulating water bath $\left(15^{\circ} \mathrm{C}\right)$. Remove oxygen from the water by bubbling nitrogen through it for 20 minutes. This prevents the oxygen contamination through the flow circuit.

3. Maintain the temperature of the drive syringes and the observation cell at $15{ }^{\circ} \mathrm{C}$ by connecting the water bath housing of the stopped-flow to the circulating bath.

4. In the Control Pane/ window of the Pro-Data control software select Photodiode Array, External trigger (to activate stopped-flow operation), and Logarithmic scale.

5. Launch the Pro-Data viewer software and define the directory where the data files will be stored.

6. Replace the solution of reservoir syringes $\mathrm{C}$ and $\mathrm{F}$ with anaerobic $100 \mathrm{mM}$ potassium phosphate buffer ( $\mathrm{pH} 7.5$ ). Turn the $\mathrm{C}$ and $\mathrm{F}$ valves to the "drive" position. Empty the stop syringe by clicking Empty in the Pro-Data control software. Push the buffer from both drive syringes through the flow circuit by manually raising the corresponding drive ram five times in total (click Empty before each movement of the ram).

7. In order to ensure that glucose oxidase has been removed completely from the flow circuit, perform step 6.6 four times in total.

8. Click on Baseline in the Pro-Data control software.

9. Mix $100 \mu \mathrm{L}$ of enzyme stock solution with $1100 \mu \mathrm{L}$ of anaerobic $100 \mathrm{mM}$ potassium phosphate buffer (final concentration after mixing in the stopped flow of $15 \mu \mathrm{M}$ ).

10. Replace the solution of reservoir syringe $F$ with the enzyme solution. Turn the $F$ valve to the "drive" position. Empty the stop syringe by clicking Empty in the Pro-Data control software. Push the enzyme solution from drive syringe $F$ through the flow circuit by manually raising the corresponding drive ram. Perform this three times in total. In the Pro-Data control software, set the acquisition time to $60 \mathrm{~s}$.

11. Ensure that both drive syringes are filled with their corresponding solutions and the drive rams are in contact with the drive syringe pistons. Turn both valves to the "drive" position and click Acquire in the Pro-Data control software to perform the drive. Repeat this step two times more to obtain the data in triplicate. This yields spectra of the oxidized form of the enzyme.

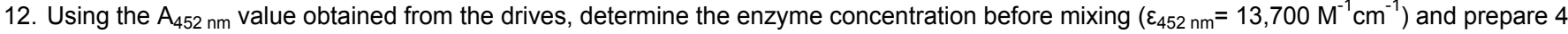
$\mathrm{mL}$ of a NADPH solution 1.5-fold more concentrated.

13. Replace the solution of reservoir syringe $C$ with the NADPH solution and empty and refill the stop syringe three times as described in 6.10

14. Repeat step 6.11. This yields spectra during the reduction of the enzyme.

\section{Oxidative Half-reaction: Monitoring Flavin Oxidation}

1. Prepare the stopped-flow instrument and the Pro-Data control software for double mixing mode following the manufacturer's protocol.

2. Repeat steps 6.2-6.5.

3. Replace the solution in the four reservoir syringes with anaerobic $100 \mathrm{mM}$ potassium phosphate buffer ( $\mathrm{pH}$ 7.5). Turn the $\mathrm{F}$ valve to the "drive" position. Empty the stop syringe by clicking Empty in the Pro-Data control software. Push the buffer from the drive syringe $\mathrm{F}$ through the flow circuit by manually raising the corresponding drive ram. Perform this step five times in total with each drive syringe.

4. Perform step 7.3 four times in total to completely remove the contents from the flow circuit. 
5. Click Baseline in the Pro-Data control software.

6. Mix $200 \mu \mathrm{L}$ of enzyme stock solution with $1000 \mu \mathrm{L}$ of anaerobic $100 \mathrm{mM}$ potassium phosphate buffer (final concentration after mixing in the stopped flow of $15 \mu \mathrm{M}$ ).

7. Replace the solution of reservoir syringe A with the enzyme solution. Turn the A valve to the "drive" position. Empty the stop syringe by clicking Empty in the Pro-Data control software. Push the enzyme solution from drive syringe A through the flow circuit by manually raising the corresponding drive ram. Perform this step three times in total. In the Pro-Data control software, set the delay time to $0.5 \mathrm{~s}$ and acquisition time to $60 \mathrm{~s}$.

8. Ensure that all drive syringes are filled with their corresponding solutions and the drive rams are in contact with the drive syringe pistons. Turn all valves to the "drive" position and click Acquire in the Pro-Data control software to perform the drive. Repeat this step two more times to obtain the data in triplicate. This yields spectra of the oxidized form of the enzyme.

9. Replace the solution of reservoir syringe $B$ with an NADPH solution 1.5-fold more concentrated than the enzyme solution in syringe A. Turn the $B$ valve to the "drive" position and empty and refill the stop syringe three times as described in 7.7.

10. Replace the solution of reservoir syringe $C$ with oxygenated buffer. Turn the $C$ valve to the "drive" position and empty and refill the stop syringe three times as described in 7.7. In the Pro-Data control software, set the delay and acquisition time at 15 and $900 \mathrm{~s}$, respectively.

11. Repeat step 7.8. This yields spectra during the reoxidation of the fully reduced enzyme.

\section{Data Analysis}

1. Launch the Pro-Data converter software. Click on the Options icon and select Save in ProDataCSV.

2. Open the directory where the data files were stored. Drag the files to the APL Pro-Data converter window. The data will be saved automatically as CSV format files in the same directory.

3. Open the CSV format files using Microsoft Excel (Microsoft, Redmond, WA, USA). Normalize the baseline of the stopped-flow traces by subtracting the corresponding absorption at $700 \mathrm{~nm}$.

4. Analyze the corrected traces using KaleidaGraph (Synergy Software, Reading, PA) by fitting the plot of the absorbance at the corresponding maximum versus time to the appropriate exponential function. As shown in Figure 2B, the absorbance at $452 \mathrm{~nm}$ was plotted as a function of time in order to determine the rate of flavin reduction after mixing the enzyme and NADPH in the stopped-flow instrument. In this case, the best fit of the data was obtained using a double exponential equation and rates of 0.65 and $0.23 \mathrm{~s}^{-1}$ were calculated for the first and second phase of the reduction, respectively. To determine the rate of formation of the C4a-hydroperoxyflavin intermediate and the reoxidation after reacting the reduced enzyme with oxygen, we analyzed the stopped-flow traces at 380 and $452 \mathrm{~nm}$, respectively (Figure 3B). Using a single exponential equation, we calculated rates of 1.4 and $0.006 \mathrm{~s}^{-1}$ for the first and second step of the oxidative half-reaction, respectively.

\section{Representative Results}

The results from the experiments described in the previous sections show how the reductive half-reaction of $A f$ SidA can be monitored by measuring the changes in the absorbance at $452 \mathrm{~nm}$, which correspond to the changes in the redox state of the flavin. The rate of this step can be determined by fitting the data to the appropriate equation (Figure 2; Step 8.4). The reduction rate obtained $\left(0.65 \mathrm{~s}^{-1}\right)$ is similar to the $\mathrm{k}_{\mathrm{cat}}$ value calculated with steady-state experiments ${ }^{2}$, suggesting that the reduction is the rate-determining step of the reaction. Taking advantage of the double mixing mode of the stopped-flow, the rate of oxidation and the intermediates in this half-reaction can be determined (Figure 3; Step 8.4). In the reaction catalyzed by Af SidA, the C4a-hydroperoxyflavin is clearly detected $\left(\lambda_{\max }\right.$ of $\left.380 \mathrm{~nm}\right)$, and the rate of formation and decay can be calculated. The slow rate of the reoxidation obtained $\left(0.006 \mathrm{~s}^{-1}\right)$ indicates that the $\mathrm{C} 4 \mathrm{a}$-hydroperoxyflavin is very stable in absence the ornithine.

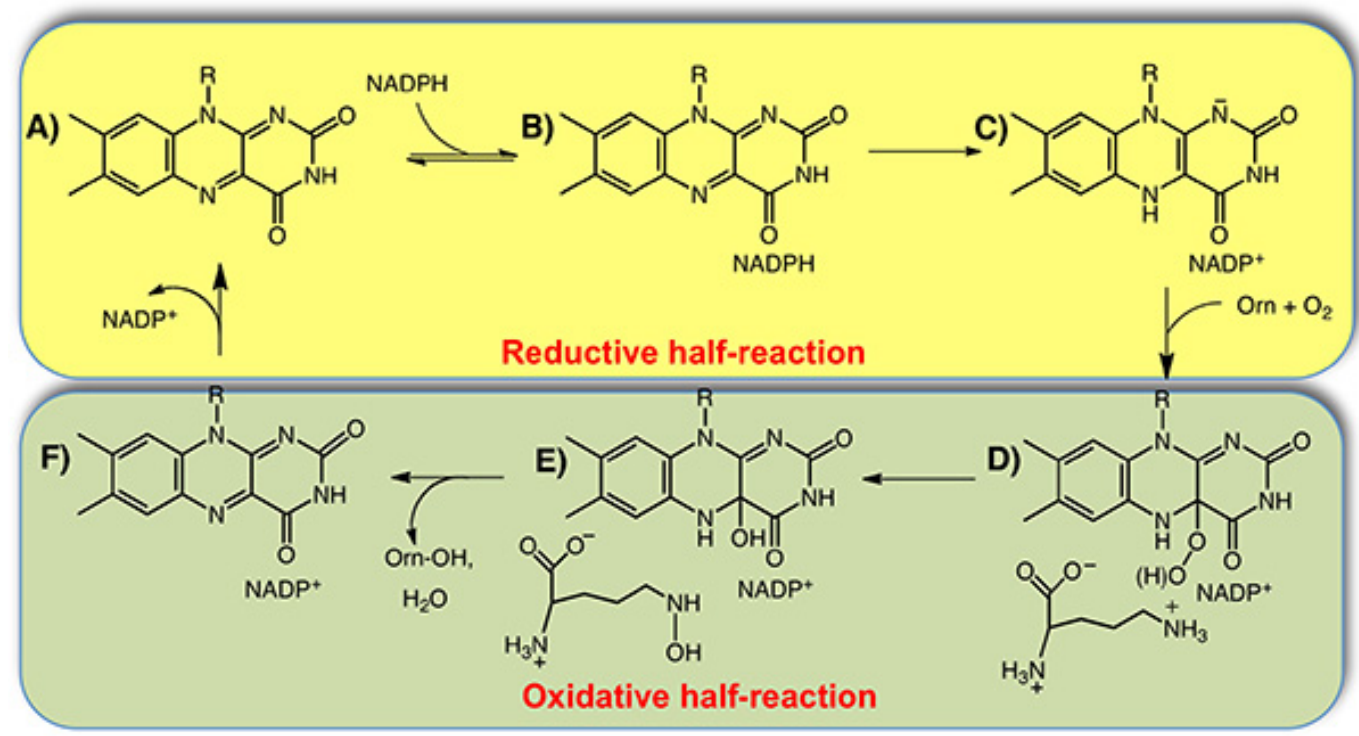

Scheme 1. Mechanism of Af SidA. The isoallozaxine ring of the FAD cofactor is shown. The oxidized flavin (A) binds to NADPH (B) and reacts to form reduced flavin and $\mathrm{NADP}^{+}(\mathrm{C})$. After reaction with molecular oxygen and binding of ornithine, the C4a-hydroperoxyflavin is formed (D). This is the hydroxylating species. After hydroxylation of ornithine, the hydroxyflavin (E) must be dehydrated to form the oxidized enzyme. NADP ${ }^{+}$ remains bound throughout the catalytic cycle and is the last product to be released $(F)$. 

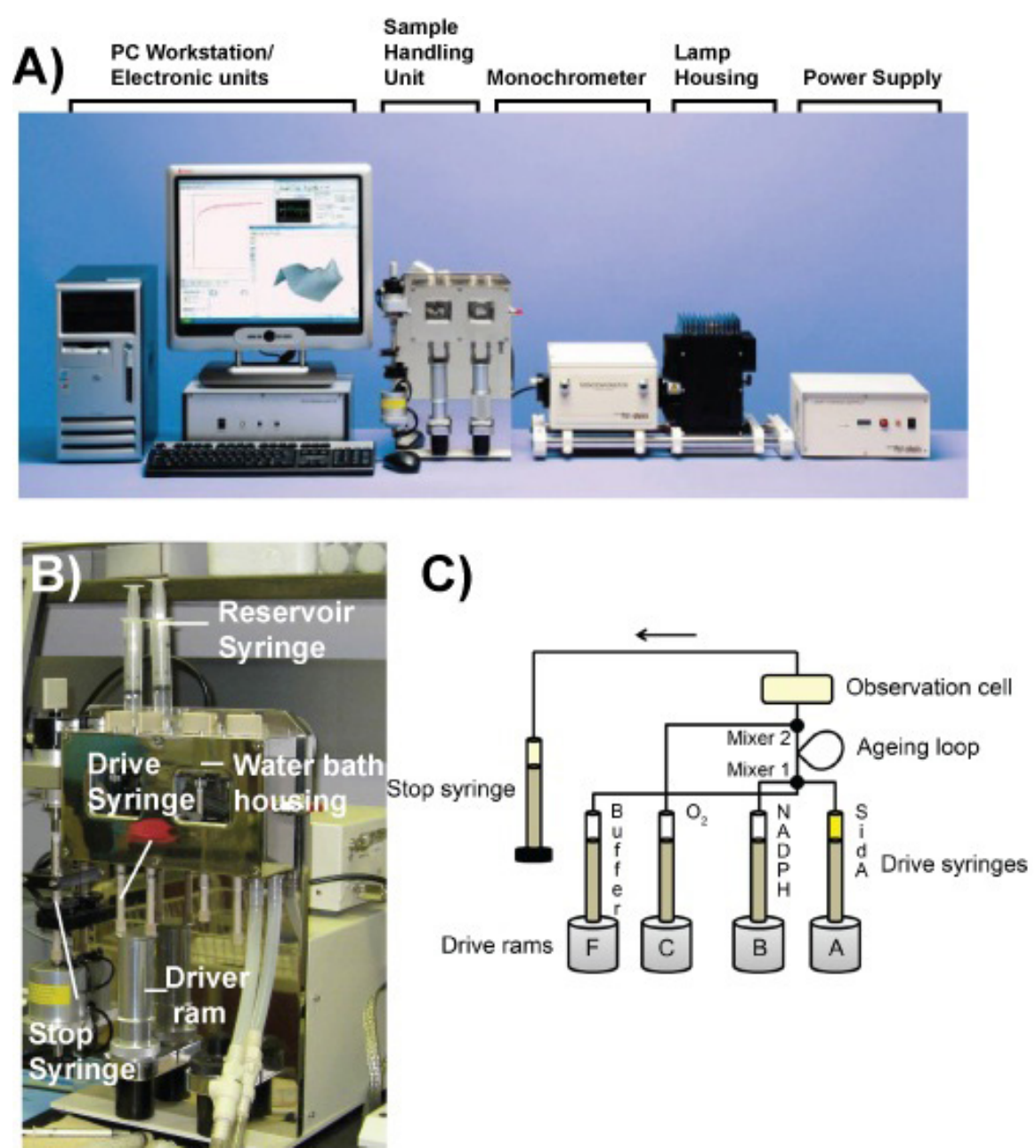

Figure 1. The stopped-flow instrument. A) Picture of the components of the Applied Photophysics SX20 stopped-flow spectrophotometer. B) Picture of the sample handling unit. C) Scheme of the flow circuit in double mixing mode.
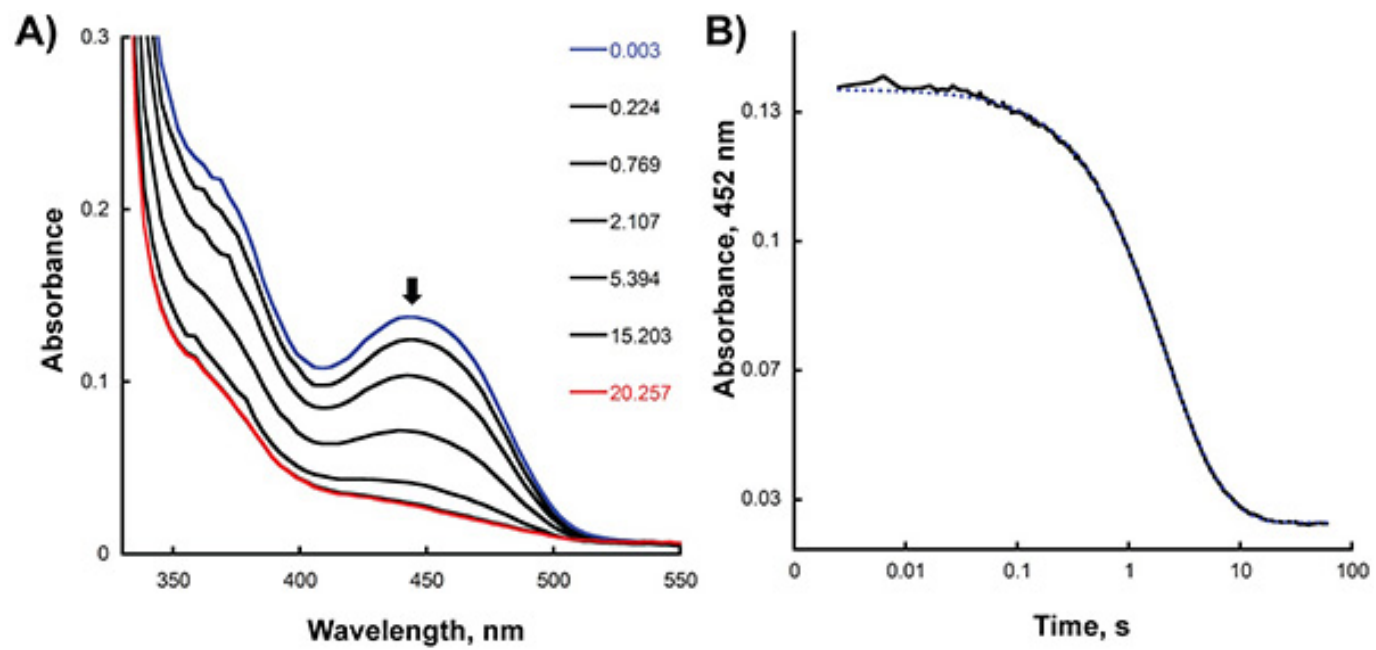

Figure 2. Anaerobic reduction of SidA with NADPH in the stopped-flow instrument. A) Spectral changes recorded after mixing equal volumes of $30 \mu \mathrm{M}$ SidA and $45 \mu \mathrm{M}$ NADPH. The first spectrum (oxidized SidA) and last spectrum (fully reduced SidA) is highlighted in blue and red, respectively. B) Absorbance trace at $452 \mathrm{~nm}$ recorded as a function of time. 


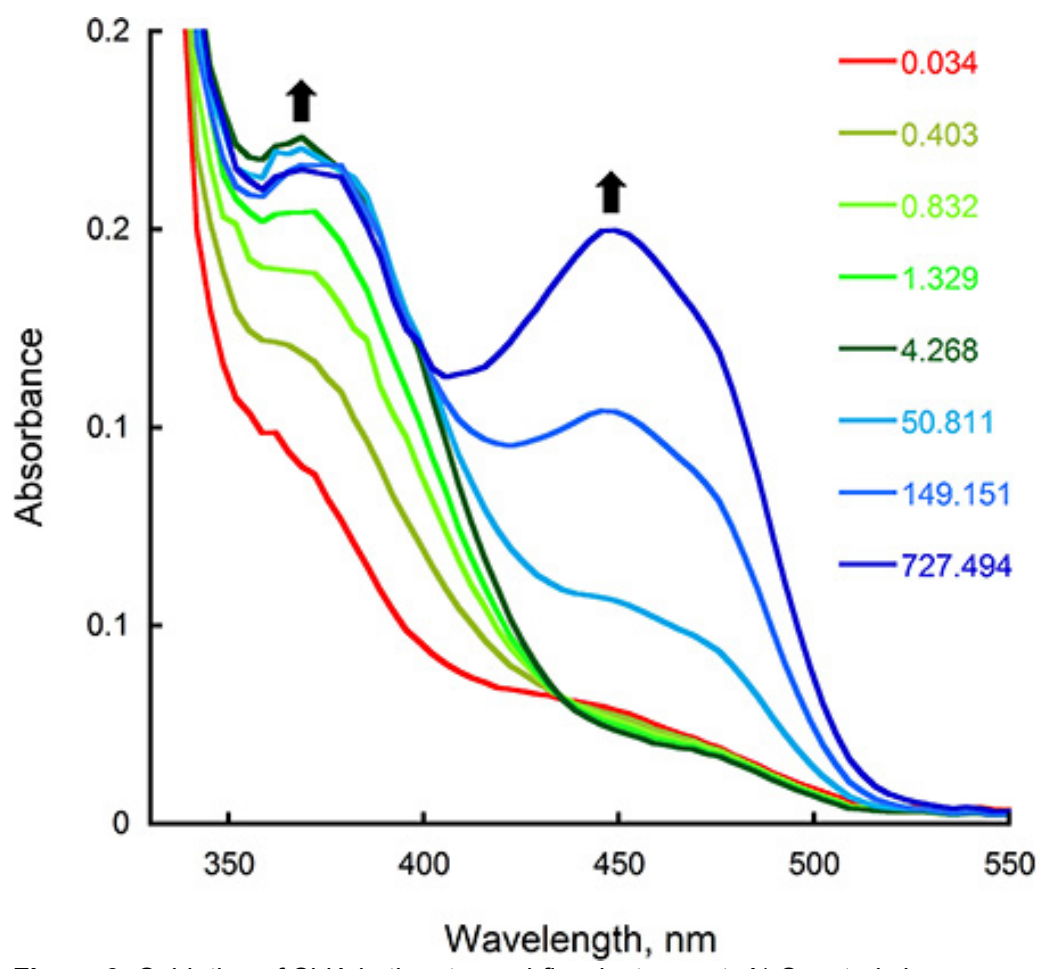

Figure 3. Oxidation of SidA in the stopped-flow instrument. A) Spectral changes recorded after mixing equal volumes of the fully reduced SidA and oxygenated buffer. The final concentrations were $15 \mu \mathrm{M}$ SidA, $22.5 \mu \mathrm{M} \mathrm{NADPH}$, and $0.95 \mathrm{mM}$ oxygen. The spectrum recorded at 0.034 , 4.268, and $727.494 \mathrm{~s}$ corresponds to the fully reduced enzyme, the C4a-hydroperoxyflavin intermediate $\left(\lambda_{\max }\right.$ of $\left.380 \mathrm{~nm}\right)$, and the oxidized enzyme $\left(\lambda_{\max }\right.$ of $\left.450 \mathrm{~nm}\right)$, respectively. B) Absorbance trace at 382 and $452 \mathrm{~nm}$ recorded as a function of time.

\section{Discussion}

Enzymes that catalyze redox reactions usually contain cofactors such as hemes and flavins that undergo significant absorbance changes during the catalytic cycle. The oxidized form of the flavin presents absorbance maxima at $\sim 360$ and $450 \mathrm{~nm}$, and its reduction is typically monitored by following the absorbance decrease at $450 \mathrm{~nm}^{7}$. In general, some transient intermediates are present but form and decay too fast to be measured in regular spectrophotometers. Using the Applied Photophysics SX20 stopped-flow spectrophotometer (or similar instruments), it is possible to measure absorbance changes in the millisecond time scale (dead-time, $2 \mathrm{~ms}$ ). Here we studied the reductive and oxidative half-reactions of the flavin-dependent monooxygenase Af SidA, serving as a model. The rate of hydride transfer was determined by measuring the change of absorbance at $452 \mathrm{~nm}$ after mixing the enzyme with NADPH under anaerobic conditions. Subsequently, taking advantage of the double mixing mode of the stopped-flow instrument, the enzyme was first reacted with NADPH, until full reduction was achieved, then the reduced enzyme-NADP ${ }^{+}$complex was mixed with oxygen. Following this procedure, it is possible to detect transient oxygenated flavin intermediates and to measure rates of formation and decay. The identification of these intermediates provides experimental data about the nature of the reacting species in catalysis. In the case of Af SidA, the formation of the C4a-hydroperoxyflavin (typically monitored at 370-380 $\mathrm{nm}$ ), which is the hydroxylating species. In addition, measuring the rate constant of each step allows one to obtain information about the rate-determining step of the reaction and help to elucidate the kinetic and chemical mechanisms of the enzyme.

In general, similar approaches can be used for other flavoenzymes, or proteins for which absorbance changes occurred, such as proteins that contain heme, pyridoxal-phosphate, or non-heme iron ${ }^{8-10}$. A limitation to this method is that large amounts of purified enzyme are required, but this can be overcome by using an expression system with high yields. One determines the optimal protein concentration for recording spectra by using enough protein so that a strong enough signal can be observed, but not too much so that enzyme is not wasted. Typically, the lowest enzyme concentration for flavin-containing enzymes used in stopped-flow experiments is $6-10 \mu \mathrm{M}$ (after mixing) and is determined using the corresponding molar extinction coefficient of the enzyme. In the case of Af SidA, the percentage of the enzyme bound FAD is 50-65\% ${ }^{2}$. Apoprotein is regarded as inactive in these experiments because a bound FAD cofactor is necessary for catalysis. Another possible limitation to this method is if processes in an enzyme occur faster than $2 \mathrm{~ms}$ (dead time of the stopped-flow) they will not be observed, but there are reported strategies where rates can be decreased to overcome this issue. One example for this includes using a high $\mathrm{NaCl}$ concentration in the reaction of a ferredoxin-NADP ${ }^{+}$reductase ${ }^{11}$. The scrubbing of oxygen from the flow circuit of the stopped-flow is often a tricky step in this experiment and requires special attention. The glucose oxidase-glucose system described here is used successfully in most laboratories as it is an effective and inexpensive method. However, there are some drawbacks which include the production of $\mathrm{H}_{2} \mathrm{O}_{2}$ and for some applications other alternatives as the protocatechuate dioxygenase-protocatechuate system should be considered ${ }^{12}$. The utilization of an anaerobic glove box makes it easier to ensure anaerobic conditions, but is not essential. Oxygen must be removed from the flow circuit of the stopped-flow as we want the enzyme to be reduced in the absence of oxygen or react with oxygen at concentrations that we specify. Although the stopped-flow is in the glove box, there is oxygen in the flow circuit if we used aerobic buffers in previous experiments. In addition to absorbance measurements, fluorescence and circular dichroism assays can be performed in the Applied Photophysics SX20 stopped-flow spectrophotometer with the corresponding accessories. 


\section{Disclosures}

We have nothing to disclose.

\section{Acknowledgements}

Research supported by NSF award MCB-1021384.

\section{References}

1. Hissen, A.H., et al. The Aspergillus fumigatus siderophore biosynthetic gene sidA, encoding L-ornithine N5-oxygenase, is required for virulence. Infect. Immun. 73 (9), 5493-5503 (2005).

2. Chocklett, S.W. \& Sobrado, P. Aspergillus fumigatus SidA is a highly specific ornithine hydroxylase with bound flavin cofactor. Biochemistry. 49 (31), 6777-6783 (2010).

3. Mayfield, J.A., et al. Comprehensive spectroscopic, steady state, and transient kinetic studies of a representative siderophore-associated flavin monooxygenase. J. Biol. Chem. 285 (40), 30375-30388 (2010).

4. Fierke, C.A. \& Hammes, G.G. Transient kinetic approaches to enzyme mechanisms. In: Contemporary enzyme kinetics and mechanism. Elsevier, USA, (2009).

5. Palfey, B.A. \& McDonald, C.A. Control of catalysis in flavin-dependent monooxygenases. Arch. Biochem. Biophys. 493 (1), 26 (2010).

6. van Berkel, W.J.H., Kamerbeek, N.M., \& Fraaije, M.W. Flavoprotein monooxygenases, a diverse class of oxidative biocatalysts. J. Biotechnol. 124 (4), 670-689 (2006).

7. Chapman, S.K. \& Reid, G.A. In: Flavoprotein Protocols. Humana Press, Totowa, USA, (1999).

8. Sobrado, P. \& Fitzpatrick, P.F. Solvent and primary deuterium isotope effects show that lactate $\mathrm{CH}$ and $\mathrm{OH}$ bond cleavage are concerted in Y254F flavocytochrome $b_{2}$, consistent with a hydride transfer mechanism. Biochemistry. 42, 15208-15214 (2003).

9. Kumar, S., Gawandi, V.B., Capito, N., \& Phillips, R.S. Substituent effects on the reaction of \&beta;-benzoylalanines with Pseudomonas fluorescens kynureninase. Biochemistry. 49 (36), 7909-7913 (2010).

10. Yun, D., García-Serres, R., Chicalese, B.M., An, Y.H., Huynh, B.H., \& Bollinger, J.M.J. ( $\mu$-1,2-Peroxo)diiron(III/III) complex as a precursor to the diiron(III/IV) intermediate $\mathrm{X}$ in the assembly of the iron-radical cofactor of ribonucleotide reductase from mouse. Biochemistry. 46 (7), 1925-32 (2007).

11. Pennati, A., Zanetti, G., Aliverti, A., \& Gadda, G. Effect of salt and pH on the reductive half-reaction of Mycobacterium tuberculosis FprA with NADPH Biochemistry. Biochemistry. 47 (11), 3418-3425 (2008).

12. Patil, P.V. \& Allou, D.P. The use of protocatechuate dioxygenase for maintaining anaerobic conditions in biochemical experiments. Analytical Biochemistry. 286 (2), 187-192 (2000). 